\title{
Clinical Features and Echocardiographic Findings in Children with Hypertrophic Cardiomyopathy
}

\author{
Blesneac Cristina ${ }^{1}$, Benedek Theodora², Togănel Rodica ${ }^{1}$, Benedek ${ }^{2}$ \\ 1 Department M4, IIIrd Pediatric Discipline, University of Medicine and Pharmacy, Tîrgu Mureș, Romania \\ ${ }^{2}$ Clinic of Cardiology, Department M3, University of Medicine and Pharmacy, Tîrgu Mureș, Romania
}

\begin{abstract}
Background: Hypertrophic cardiomyopathy, one of the most common inherited cardiomyopathies, is a heterogeneous disease resulting from sarcomeric protein mutations, with an incidence in the adult population of 1:500. Current information on the epidemiology and outcomes of this disease in children is limited.

Methods: Thirty-four children diagnosed with hypertrophic cardiomyopathy in the Pediatric Cardiology Department from Tîrgu Mureș were evaluated concerning familial and personal history, clinical, paraclinical and therapeutic aspects. Hypertrophic cardiomyopathy was defined by the presence of a hypertrophied, non-dilated ventricle, in the absence of a cardiac or systemic disease that could produce ventricular hypertrophy. Results: The youngest diagnosed child was a neonate, a total of 10 patients being diagnosed until 1 year of age. In 6 cases a positive familial history was found. Noonan syndrome was found in 2 cases. Only 21 patients were symptomatic, the predominant symptoms being shortness of breath on exertion with exercise limitations. Left ventricular outflow tract obstruction was present in 21 cases (61.7\%). Twenty-four patients were on $\beta$-blocking therapy, while 4 patients underwent septal myectomy.

Conclusions: Hypertrophic cardiomyopathy is a heterogeneous disorder in terms of evolution, age of onset, type and extent of hypertrophy, and the risk of sudden death. It can affect children of any age. There is a need for a complex evaluation, including familial and personal anamnesis, clinical examination, electrocardiogram and echocardiography of all patients. It is highly important to develop screening strategies, including genetic testing, for an early diagnosis, especially in asymptomatic patients with a positive familial background.
\end{abstract}

Keywords: hypertrophic, cardiomyopathy, children

Received: 13 July 2013

\section{Introduction}

Hypertrophic cardiomyopathy (HCM), a heterogeneous disease resulting from sarcomeric contractile protein mutations, is one of the most common genetic cardiac disorders [1]. The reported prevalence of the phenotypically expressed HCM in the adult general population is about 1:500 [1]. HCM represents the most common cause of sudden cardiac death in young adults [2]. It is characterized by left-ventricular hypertrophy in the absence of hypertension and valve disease [3].

Current information on the epidemiology and outcomes of this disease in children is limited. Registries from the US and Australia show a prevalence varying between 0.47 and 1.24:100,000 inhabitants and an occurrence of nearly $25 \%$ among all types of cardiomyopathies $[4,5]$, with a peak incidence during the first year of life [5].

$\mathrm{HCM}$ is a highly penetrant genetic disease, in most adults and adolescents being frequently inherited as an autosomal dominant trait caused by mutations in the genes encoding a range of cardiac sarcomere proteins $[6,7,8,9,10]$. In contrast, a familial disease is reported in less than $20 \%$ of $\mathrm{HCM}$ cases affecting infants and children $[11,12]$. In some young patients, left ventricular hypertrophy is associated with inborn errors of metabolism, neuromuscular disease,

Correspondence to: Theodora Benedek

E-mail: hintea_teodora@yahoo.com such as Friedreich's ataxia, and congenital malformation syndromes, such as Noonan and LEOPARD syndromes $[13,14]$, but recent studies suggest that these conditions account for $<10 \%$ of cases $[11,12]$. The leading genetic cause of infantile HCM is Noonan syndrome (NS) [15], the incidence of this disease being estimated to be between 1 in 1000 and 1 in 2500 live births $[16,17]$. HCM is reported in $20 \%$ to $30 \%$ of all NS cases $[15,17,18]$.

HCM may be initially suspected because of a heart murmur, positive family history, new symptoms or abnormal ECG pattern showing left ventricular hypertrophy and abnormal Q waves [3]. Physical examination may not identify this disorder, given that most patients do not have left ventricular outflow tract obstruction and most of the physical findings (systolic murmur, bifid arterial pulse) are limited to patients with outflow gradients. Echocardiography plays a pivotal role in detecting the disease, by imaging the hypertrophied, but non-dilated left-ventricular chamber, in the absence of another cardiac or systemic disease (hypertension or aortic stenosis).

Reviews based on institutional experience have suggested a poor outcome for childhood HCM, outcomes in cases presenting in the first year of life being substantially worse than when HCM is diagnosed after the first year of life $[11,12,19,20]$. Mortality rates in HCM presenting in childhood are higher (annual rate 4.6-5.8\%) [21,22] than in adult life (1-4\%) [23,24]. 


\section{Methods}

\section{Study patients}

The database at the IInd Pediatric Cardiology Department from the County Emergency Clinical Hospital Tîrgu Mures was reviewed to identify patients diagnosed with HCM. All patients under 18 years of age diagnosed with HCM were included in the study. Original patient records and echocardiographic data were re-examined. Children with cardiac hypertrophy secondary to cardiac or systemic diseases were excluded.

\section{Measurements}

Medical records were reviewed to determine the patients' age at the time of diagnosis, the presence or absence of symptoms (syncope, chest pain, shortness of breath on exertion, exercise limitations, palpitations) and familial history of HCM or sudden cardiac death.

Data from 12-lead electrocardiograms (ECGs), ambulatory ECGs and echocardiography were collected. Treatment regimens were also noted. In this study, the patients with NS were diagnosed clinically by phenotypic features. Familial HCM was considered present if there was an affected first- or second-degree relative.

HCM was defined by unexplained septal and/or free wall hypertrophy $(Z$ score $>2$ based on body surface area for either) [25]. Asymmetric septal hypertrophy was defined as a septal to posterior left-ventricular free wall ratio greater than 1.5 [26]. Left ventricular outflow tract obstruction was defined as anatomic narrowing, together with a measured peak systolic gradient $>15 \mathrm{mmHg}$ through echocardiography [12].

\section{Statistical analysis}

Statistical analysis was performed using Graph Pad Prism. Quantitative variables were expressed by median and range, after they were initially subjected to KolmogorovSmirnov concordance test. Student's paired t test was used to assess differences between median of continuous variables (expressed as mean $\pm \mathrm{SD}$ ), while categorical variables were expressed as no (\%). All tests were interpreted to the significance threshold value of $\mathrm{p}=0.05$, and statistical significance was considered for $\mathrm{p}$ values smaller than the significance threshold value.

\section{Results}

A total of 34 patients with HCM were identified. Table I summarizes the main characteristics of the studied subjects. In the studied population, 21 patients $(61.8 \%)$ were male and 13 female. The median age at diagnosis was 2 years (range 0.1 to 17 years). The youngest one was a neonate, a total of 10 patients (29.4\%) being diagnosed until 1 year of age.

Familial HCM was found in 6 subjects (17.6\%), all with at least one affected adult family member. Among them, three subjects $(50 \%)$ were diagnosed before 1 year of age.
NS was present in 2 patients (5.8\%). Neither one had positive familial history for HCM. In 2 other cases, based on the phenotype, a genetic syndrome was suspected, but the clinical aspect of the subjects was not highly specific to a certain syndrome, and due to lack of advanced methods in genetic testing, a specific genetic syndrome was not established.

Presenting symptoms in 14 cases (41.1\%) were shortness of breath on exertion and exercise limitations; 6 subjects (17.6\%) had palpitations associated with exercise limitations; syncope was found in 1 case; the remaining subjects $(\mathrm{n}=13,38.2 \%)$ were diagnosed after being screened for a cardiac murmur or non-specific symptoms. There was only one case of resuscitated cardiac arrest.

ECG was available in 30 patients, in 28 subjects (93\%) left ventricular hypertrophy was found; ST segment changes were found in 9 cases (26.4\%); left axis deviation was found in 14 subjects (41.1\%); left atrial enlargement was found in 7 cases $(20.5 \%)$. Arrhythmias were identified in 6 cases (17.6\%) (on 12-lead ECGs and/or 24-hour ECG monitoring): 2 subjects presented ventricular tachycardia, 2 subjects - atrial tachycardia, and 2 subjects - premature atrial contractions.

Asymmetric septal hypertrophy was found in 15 subjects (44.1\%), and concentric left ventricular hypertrophy was present in 19 patients (55.9\%). The median septal to posterior left-ventricular free wall ratio was 1.42 (range 0.87 to 5.73 ). Both children with NS had asymmetric septal hypertrophy. The mean fractional shortening in the studied subjects was $43.73 \pm 11.32 \%$. Right ventricular hypertrophy was found in 2 cases $(5.88 \%)$.

Left ventricular outflow tract obstruction with a resting gradient was present in 21 cases $(61.7 \%)$ with a median peak instantaneous gradient of $116 \mathrm{mmHg}$ (range 21 to $198 \mathrm{mmHg}$ ), among them 4 subjects (19\%) presented left ventricular midcavity obstruction in association with an outflow gradient. In one case there was isolated left ventricular midcavity obstruction.

Four patients underwent septal myectomy to relieve left ventricular outflow tract obstruction. The median age at the time of the procedure was 5.4 years (range 5 to 8 years), a median of 4.7 years (range 1 to 5.5 years) after diagnosis. The mean peak echocardiographic gradient before surgery

Table I. Main characteristics of the study population

\begin{tabular}{lc}
\hline Male/female, $\mathrm{n}(\%)$ & $21 / 13(61.8 / 38.2)$ \\
Age $<1$ year at time of diagnosis, $\mathrm{n}(\%)$ & $10(31.2)$ \\
Noonan syndrome, $\mathrm{n}(\%)$ & $2(5.8)$ \\
Familial hypertrophic cardiomyopathy, $\mathrm{n}(\%)$ & $6(17.6)$ \\
Morphology of left ventricular involvement, $\mathrm{n}(\%)$ & \\
$\quad$ Asymmetric septal hypertrophy & $15(44.1)$ \\
$\quad$ Concentric left ventricular hypertrophy & $19(55.9)$ \\
Fractional shortening & \\
$\quad$ Mean & 43.73 \\
$\quad$ SD & 11.32 \\
Left ventricular outflow obstruction, $\mathrm{n}(\%)$ & $21(61.7)$ \\
Surgical left ventricular myectomy, $\mathrm{n}(\%)$ & $4(11.7)$
\end{tabular}


p-0.02

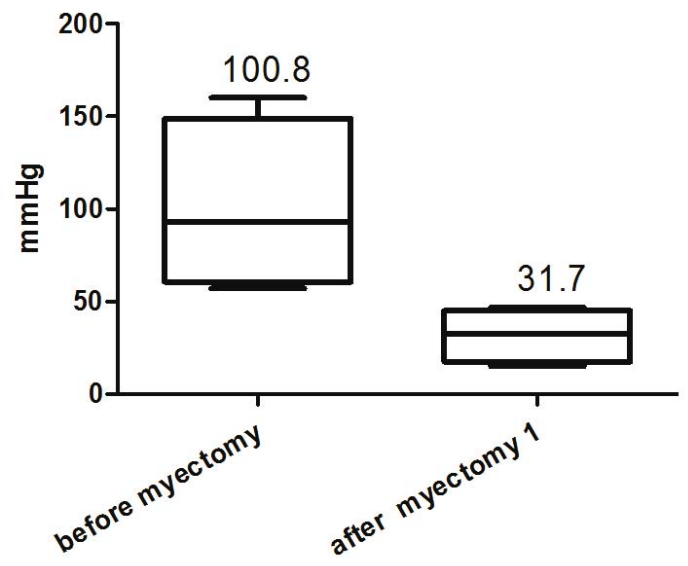

Fig. 1. The mean peak echocardiographic gradient before surgery and at first month follow-up

and at first month follow-up was $100.8 \pm 46.59 \mathrm{mmHg}$ and $31.7 \pm 14.38 \mathrm{mmHg}$, respectively $(\mathrm{p}=0.02)$ (Figure 1). The mean peak echocardiographic gradient at the latest follow-up was $66.75 \pm 27.45 \mathrm{mmHg}(\mathrm{p}=0.01)$ (Figure 2). In 2 cases, at the latest follow-up the residual gradient was significant.

Twenty-five patients $(73.5 \%)$ were receiving regular medication: a $\beta$-blocking agent $(n=24)$ or a calcium channel blocker $(\mathrm{n}=1)$. Among them, 2 patients received therapy with amiodarone for associated arrhythmia.

\section{Discussions}

HCM is a heterogeneous disorder in terms of evolution, age of onset, type and extent of hypertrophy, and the risk of sudden death. It can affect children of any age.

Due to technological advances in pediatric cardiology and echocardiography, the diagnosis of HCM became possible very early in life. As shown in our study, the youngest patient diagnosed with this disorder was a neonate, with almost $30 \%$ of the patients being diagnosed until 1 year of age. The median age at time of diagnosis was 2 years.

Studies in the specialized literature report the presence of a familial disease in infants and children in less than $20 \%$ of cases $[11,12]$. In our study, familial HCM was found in $17.6 \%$ of subjects. Because in most adults and adolescents HCM is frequently inherited as an autosomal dominant trait caused by mutations in the genes encoding a range of cardiac sarcomere proteins $[6,7,8,9,10]$, these findings strengthen the importance of clinical screening of all first-degree relatives of any patient diagnosed with HCM. Genetic testing for mutations in the genes encoding cardiac sarcomere proteins was not available due to lack of advanced methods in genetic testing in our center.

Most clinical reviews suggest that the leading cause of infantile HCM is NS [15], this affection being reported in $20 \%$ to $30 \%$ of all NS cases $[15,17,18]$. In our study, there were only 2 cases of NS (5.8\%), this fact might be explained by the incidence of the disease $[16,17]$ and the

\section{$\mathbf{P}-0.01$}

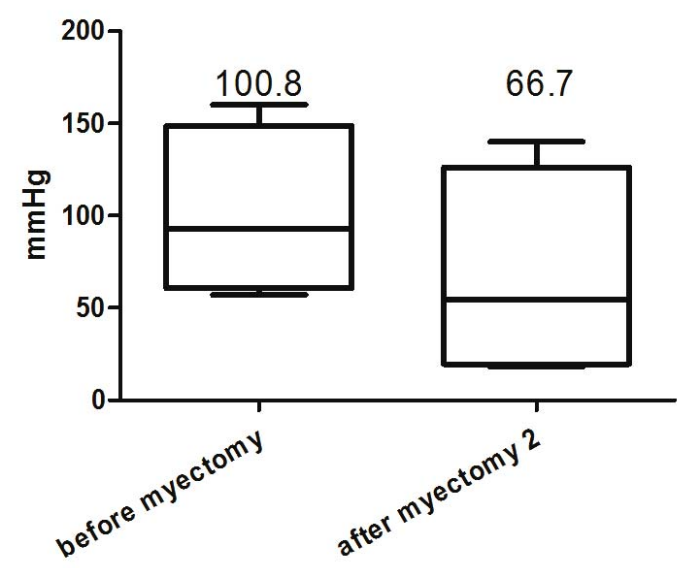

Fig. 2. The mean peak echocardiographic gradient before surgery and at latest follow-up

difficulty in recognizing the phenotype in this age group [27].

Regarding presenting symptoms, $38.2 \%$ of cases were diagnosed only after being screened for a cardiac murmur or non-specific symptoms, this finding strengthening once again the importance of clinical screening of all first-degree relatives of any patient diagnosed with HCM, even if they are asymptomatic.

The pattern of left ventricular hypertrophy is variable. Based on septal to posterior left-ventricular free wall ratio, we found only a slight difference between asymmetric septal hypertrophy and concentric left ventricular hypertrophy ( $44.1 \%$ vs. $55.9 \%)$, international studies reporting varied proportions of the two forms of HCM [11,12,27,28].

Left ventricular outflow tract obstruction with a resting gradient was present in $61 \%$ of the subjects; left ventricular midcavity obstruction was associated in 4 cases. These findings are consistent with the results reported in international studies $[12,28]$.

In adults with HCM, surgical relief of left ventricular outflow tract obstruction has been shown to be effective in reducing symptoms $[29,30]$. In the present study, as in other reported pediatric series, there was a significant reduction in the gradient [31,32]. In two cases, echocardiography performed at the latest follow-up showed a significant residual gradient in the left-ventricular outflow tract, demonstrating the fact that septal hypertrophy has the potential of reappearing.

\section{Conclusion}

Hypertrophic cardiomyopathy is a heterogeneous disorder in terms of evolution, age of onset, type and extent of hypertrophy, and the risk of sudden death. It can affect children of any age, including neonates. Familial HCM was found in less than $20 \%$ of subjects. The pattern of left ventricular hypertrophy is variable. Left ventricular outflow tract obstruction is common. There is a need for a complex clinical evaluation, including familial and personal anamnesis, clini- 
cal examination, electrocardiogram and echocardiography of all patients diagnosed with hypertrophic cardiomyopathy. It is highly important to develop screening strategies, including genetic testing, for an early diagnosis, especially in asymptomatic patients with positive familial background.

\section{References}

1. Maron BJ. Hypertrophic cardiomyopathy: a systematic review. JAMA. 2002;287:1308-1320.

2. Maron BJ. Sudden death in young athletes. N Engl J Med. 2003;349:10641075.

3. Losi MA, Nistri S, Galderisi M, et al. Echocardiography in patients with hypertrophic cardiomyopathy: usefulness of old and new techniques in the diagnosis and pathophysiological assessment. Cardiovascular Ultrasound 2010;8:7.

4. Lipshultz SE, Sleeper LA, Towbin JA, et al. The incidence of pediatric cardiomyopathy in two regions of the United States. N Engl J Med 2003;348(17):1647-55. [PubMed: 12711739]

5. Nugent AW, Daubeney PE, Chondros P, et al., for the National Australian Childhood Cardiomyopathy Study. The epidemiology of childhood cardiomyopathy in Australia. N Engl J Med. 2003;348:1639-1646.

6. Ahmad F, Seidman JG, Seidman CE. The genetic basis for cardiac remodeling. Annu Rev Genomics Hum Genet. 2005;6:185-216.

7. Marian AJ, Roberts R. The molecular genetic basis for hypertrophic cardiomyopathy. J Mol Cell Cardiol. 2001;33:655-670.

8. Niimura H, Patton KK, McKenna WJ, et al. Sarcomere protein gene mutations in hypertrophic cardiomyopathy of the elderly. Circulation. 2002;105:446-451.

9. Richard P, Charron P, Carrier L, et al. Hypertrophic cardiomyopathy: distribution of disease genes, spectrum of mutations, and implications for a molecular diagnosis strategy. Circulation. 2003;107:2227-2232.

10. Seidman JG, Seidman C. The genetic basis for cardiomyopathy: from mutation identification to mechanistic paradigms. Cell. 2001;104:557567.

11. Colan SD, Lipshultz SE, Lowe AM, et al. Epidemiology and cause-specific outcome of hypertrophic cardiomyopathy in children: findings from the Pediatric Cardiomyopathy Registry. Circulation. 2007;115:773-781.

12. Nugent AW, Daubeney PE, Chondros $P$, et al. Clinical features and outcomes of childhood hypertrophic cardiomyopathy: results from a national population-based study. Circulation. 2005;112:1332-1338.

13. Schwartz ML, Cox GF, Lin AE, et al. Clinical approach to genetic cardiomyopathy in children. Circulation. 1996;94:2021-2038.

14. Maron BJ. Hypertrophic cardiomyopathy in childhood. Pediatr Clin North Am. 2004;51:1305-1346.

15. Marino B, Digilio MC, Toscano A, et al. Congenital heart disease in children with Noonan syndrome: an expanded cardiac spectrum with high prevalence of atrioventricular canal. J Pediatr 1999;135:703-6.
16. Van der Burgt I. Noonan syndrome. Orphanet J Rare Dis. 2007;2:4.

17. Burch M, Sharland M, Shinebourne E, et al. Cardiologic abnormalities in Noonan syndrome: phenotypic diagnosis and echocardiographic assessment of 118 patients. J Am Coll Cardiol. 1993;22:1189-92.

18. Allanson JE. Noonan syndrome. In: Cassidy SB, Allanson JE, editors. Management of Genetic Syndromes. Delaware: Wiley-Liss, Inc.; 2001. p. 253-68.

19. Skinner JR, Manzoor A, Hayes AM, et al. A regional study of presentation and outcome of hypertrophic cardiomyopathy in infants. Heart. 1997;77:229-33.

20. Ostman-Smith I, Wettrell G, Riesenfeld T. A cohort study of childhood hypertrophic cardiomyopathy: improved survival following high-dose beta-adrenoreceptor antagonist treatment. J Am Coll Cardiol. 1999; 34:1813-22.

21. McKenna W, Deanfield J, Faruqui A, et al. Prognosis in hypertrophic cardiomyopathy: role of age and clinical, electrocardiographic and hemodynamic features. Am J Cardiol. 1981;47:532-8.

22. Maron BJ, Henry NL, Clark CE, et al. Asymmetric septal hypertrophy in childhood. Circulation. 1976;53:9-19.

23. Seiler C, Hess OM, Schoenbeck M, et al. Long-term follow-up of medical versus surgical therapy for hypertrophic cardiomyopathy: a retrospective study. J Am Coll Cardiol. 1991;17:634-42.

24. Kofflard MJ, Waldstein DJ, Vos J, ten Cate FJ. Prognosis in hypertrophic cardiomyopathy observed in a large clinic population. Am J Cardiol. 1993;72:939-43.

25. Kampmann C, Wiethoff C, Wenzel A, et al. Normal values of M mode echocardiographic measurements of more than 2000 healthy infants and children in central Europe. Heart. 2000;83(6):667-672.

26. Rakowski H, Fulop J, Wigle ED. The role of echocardiography in the assessment of hypertrophic cardiomyopathy. Postgrad Med J. 1986;62:557-61.

27. Wilkinson J, Lowe A, Salbert B, et al. Outcomes in children with Noonan syndrome and hypertrophic cardiomyopathy: A study from the Pediatric Cardiomyopathy Registry. Am Heart J, 2012;164:442-8.

28. Yetman A, Hamilton R, Benson L, McCrindle B. Long-term outcome and prognostic determinants in children with hypertrophic cardiomyopathy. J Am Coll Cardiol. 1998;32:1943-50.

29. McCully RB, Nishimura RA, Tajik AJ, Schaff HV, Danielson GK. Extent of clinical improvement after surgical treatment of hypertrophic obstructive cardiomyopathy. Circulation. 1996;94:467-471.

30. Robbins RC, Stinson EB. Long-term results of left ventricular myotomy and myectomy for obstructive hypertrophic cardiomyopathy. J Thorac Cardiovasc Surg. 1996;111:586-594.

31. Mohr R, Schaff HV, Puga FJ, Danielson GK. Results of operation for hypertrophic obstructive cardiomyopathy in children and adults less than 40 years of age. Circulation. 1989;80(pt 1):I-191-I-196.

32. Theodoro DA, Danielson GK, Feldt RH, Anderson BJ. Hypertrophic obstructive cardiomyopathy in pediatric patients: results of surgical treatment. J Thorac Cardiovasc Surg. 1996;112:1589-1597. 